\section{Standing with a Bent Knee}

I AM currently investigating knee forces by techniques similar to those already successfully used on the hip joint ${ }^{1}$. Although this work is not yet complete, it is difficult to reconcile Dr Roberts' article ${ }^{2}$ with my observations and I should be glad of the opportunity to comment on this from an engineering viewpoint.

From the lever factors $y / x$ in Fig. 3 of the article, the reader is given to understand that the equilibrium of the knee is produced by the force in vastus crureus (of the quadriceps group) acting directly about the joint centre. But this force acts on the patella which functions rather like a pulley attached to the femur and the equilibrium of the knee in the position shown must be due to the force in the patellar ligament. While the forces are similar in magnitude, they have different lever arms and the patellar ligament lever arm should surely replace $y$ in the figure.

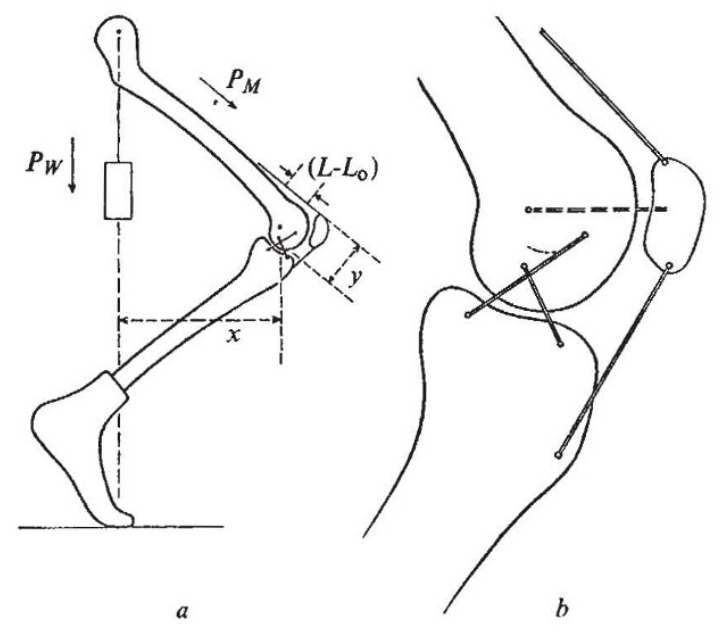

Fig. $1 a$, Diagram of model leg to show how the offsets $x$ and $y$ were measured. $b$, Detail of the pivot points at the knee. The dotted line shows the bone profile round which the posterior ligament becomes wrapped in full flexion of the knee.

The model configuration shown in Fig. 2 (shown here as Fig. 1) is a most unusual position to adopt when standing on one leg. The superimposed body weight is shown acting through the hip so that the trunk must be approximately erect to bring the centre of gravity above the hip joint; the knee is flexed at right angles while standing on the tips of the toes. I have tried to assume this posture and invited several of my colleagues to do so with almost complete lack of success except among the young and athletic who, if they could do it, found the position most uncomfortable. The natural tendency when trying to assume this position on one leg is to incline the trunk forward, thereby moving the line of gravity toward the knee to reduce the force required from the knee extensors. A good example of this forward inclination of the trunk occurs in the common and similar activity of rising from a chair without assistance from the arms. Cine film records, taken in the laboratories here, show that in extreme cases subjects bring their trunk practically horizontal when rising in this way.

Observation confirms that one-legged support is normally confined to smaller angles of knee flexion than that depicted. Yet, at very small angles of knee flexion, the line of gravity passes behind the joint, bringing the flexors into action and causing the conditions assumed by Dr Roberts to break down. It follows that the simple spring action postulated can only normally occur over quite a small range of knee angles. A further assumption implicit in the theory is that the centre of gravity will only move vertically but, in all normal activities, including balancing, horizontal movements also occur, since the centre of gravity is always free to move over a region equal to the support area of the foot. Such movements, which are likely to be as frequent as vertical movements, would require immediate compensation by the knee muscles, but would not of course affect the length of quadriceps so that spring action could be of no assistance.

In an activity such as walking, it has been known for many years that complex changes in the moment of the resultant force about the knee occur all the time ${ }^{3}$. The prop and paddle concept and the linear spring concept are surely an oversimplification of the real situation.

\section{R. D. MCLEISH}

\section{University of Manchester}

Institute of Science and Technology,

PO Box 88,

Sackville Street,

Manchester M60 1QD

Received May 4, 1971.

1 McLeish, R. D., and Charnley, J., J. Biomech., 3, 191, (1970).

2 Roberts, J. D. M., Nature, 230, 499 (1971).

${ }^{3}$ Bressler, B., and Frankel, J. P., Trans. Amer. Soc. Mech. Eng., 72, 27 (1950).

DR ROBERTs writes: In considering the lever factor, Dr McLeish looks at the force exerted by the muscle on the patella. I am looking at the forces on the femur. We are both neglecting the turning moment exerted by the compressive force between the patella and the end of the femur because the line of action of this force is offset by only a small distance from the axis of the joint. It is this force, however, that accounts for the difference between the actions of the muscle force above and below the patella.

The limb posture in Fig. 2 (shown here as Fig. 1) is less unusual than Dr McLeish maintains. The trunk does not have to be upright, if it is balanced by the free leg, neither does the line of the compressive force really have to be vertical, although in a model where a known force is to be applied at a point whose position is indeterminate it is convenient to use a hanging weight and thus to employ the vertical force of gravity.

Dr McLeish treats my article as though I had maintained that the resistance to compression is the only function to be served by the muscles acting at the knee. He thus takes no account of my treatment of the contribution of the two-joint muscles. Their action in exerting a turning moment at the hip is sufficient to account for all the situations that Dr McLeish adduces in refutation. My thesis, that it may be profitable for purposes of analysis to examine the prop and paddle actions separately, is not affected by his argument.

\section{T. D. M. ROBERTS}

Institute of Physiology,

The University,

Glasgow $W 2$

\section{Mechanism of Absorption of Phosphate from Soil by Endogone Mycorrhizas}

ECTOTROPHIC mycorrhizas have often been shown to benefit the growth of their host plants, and similar results have also been found for endotrophic mycorrhizas formed by Endogone species $^{1,2}$ which are widespread on agricultural crops and are of considerable importance. Several workers have concluded 